\title{
Síntese e processamento de cerâmicas em forno de microondas doméstico
}

\section{(Synthesis and ceramics processing by domestic microwave oven)}

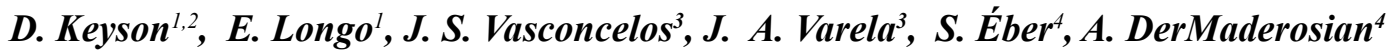 \\ ${ }^{1}$ Centro Multidisciplinar de Desenvolvimento de Materiais Cerâmicos (CMDMC), LIEC, Depto de Química \\ Universidade Federal de S. Carlos, S. Carlos, SP, Brasil 13565-905 \\ ${ }^{2}$ Laboratório de Ensino de Ciências Exatas e da Natureza - DME \\ Universidade Federal da Paraíba, PB, Brasil 58051-900 \\ ${ }^{3}$ Centro Multidisciplinar de Desenvolvimento de Materiais Cerâmicos (CMDMC), LIEC \\ Instituto de Química, Universidade Estadual Paulista, Araraquara, SP, Brasil, 14801-907 \\ ${ }^{4}$ Chemistry Department, University of the Sciences in Philadelphia, PH, EUA, 12455 \\ dawy@liec.ufscar.br
}

\begin{abstract}
Resumo
O uso de microondas no processamento e obtenção de materiais tem adquirido nos últimos anos crescente interesse por parte de diversas áreas do conhecimento como a química e a engenharia de materiais. Neste sentido, aparatos especialmente projetados têm começado a ser descritos na literatura como reatores e câmaras de processamento a microondas visando aplicação na pesquisa e na indústria. Em particular o uso de fornos de microondas domésticos em atividades científicas tem se mostrado de interesse dado uma série de novas aplicações, simplicidade e baixo custo. O presente trabalho reporta um dispositivo desenvolvido a partir de um forno de microondas doméstico, capaz de efetuar sínteses e processamentos de sólidos mesmo quando os materiais envolvidos não possuem susceptibilidade as microondas. O novo dispositivo tem sido utilizado com sucesso na síntese de óxidos metálicos e, em especial na sinterização e ordenamento de redes cristalinas de filmes finos.

Palavras-chave: microondas, processamento em microondas, cerâmicas, filmes finos.
\end{abstract}

\begin{abstract}
The use of microwaves in the processing and attainment of materials has acquired in the last years increasing interest on the part of diverse areas of the knowledge as the chemistry and the engineering of materials. In this direction, especially apparatuses have started to be described in literature as reactors and chambers of processing the microwaves aiming at application in the research and the industry. In particular the use of domestic ovens of microwaves in scientific activities it has if shown of it interests given a series of new applications, simplicity and low cost. The present work reports a device developed from an domestic microwave oven adapted, capable to effect syntheses and solid processing exactly when the involved materials do not possess susceptibility the microwaves. The new device has been used successfully in the metallic oxide synthesis and, in special in the sintering and order of crystalline lattice of thin films.
\end{abstract}

Keywords: microwaves, microwave processing, ceramics, thin films.

\section{INTRODUÇÃO}

As microondas são ondas eletromagnéticas confinadas a uma faixa do espectro que se estende de 300 a 300.000 MHz. Tão larga faixa confere a esses osciladores grande versatilidade de aplicação em função da frequiência selecionada. Radares de aviação civil, aparelhos de telefonia celular e fornos de microondas domésticos, são exemplos de dispositivos que funcionam a base de microondas e que estão presentes no cotidiano de mais de 1 bilhão de pessoas em todo mundo. Nos domínios da química e das ciências dos materiais, a aplicação da tecnologia de microondas tem despertado especial interesse na síntese de compostos orgânicas e inorgânicas e no tratamento térmico de muitos materiais em escala laboratorial e industrial [1-11]. Sob o foco dessas atividades, o forno de microondas doméstico tem deixado cada vez mais de ser visto como um mero eletrodoméstico e passado a figurar entre os equipamentos laboratoriais de utilidade na pesquisa científica [12-15].

Uma das primeiras ocorrências do uso de forno de microondas em aplicações que não o aquecimento de alimentos, remonta à década de 60 quando foi utilizado pela primeira vez para a secagem de cerâmicas vermelhas. No campo das ciências, a química analítica foi a primeira a utilizar o forno de microondas, havendo surgido na década de 70 os primeiros relatos de seu uso na preparação de amostras 
e atividades laboratoriais. Dentre os primeiros usos, podese citar a secagem de amostras, a digestão de soluções para análise elementar, a percolação para extração de substâncias e a dessorção térmica de compostos [16].

Ultimamente, no entanto, o interesse do uso de microondas em outras áreas da química e ciências afins tem decorrido da redução do número de etapas em muitas sínteses, melhoria de propriedades físicas em cerâmicas tecnológicas, e relevante economia de energia/tempo alcançada no processamento de muitos materiais [17]. Utilizando-se aparatos especialmente projetados, reatores e câmaras de processamento por microondas têm sido desenvolvidos tanto para aplicação na pesquisa quanto na indústria. Em particular, algumas simples adaptações efetuadas sobre um forno de microondas doméstico, tem rendido um novo e eficiente equipamento de utilidade na pesquisa científica. $O$ presente trabalho reporta este novo dispositivo capaz de efetuar, em regime de temperatura controlada, tanto a sínteses quanto o tratamento de sólidos, mesmo quando os materiais em questão não possuem susceptibilidade às microondas. $\mathrm{O}$ dispositivo consiste de um forno de microondas doméstico modificado, que contém uma célula rádio-susceptível inserida em sua cavidade. $\mathrm{O}$ aparato tem sido usado com sucesso na síntese de óxidos metálicos e no tratamento de outros materiais, especialmente na sinterização e ordenamento de redes cristalinas de filmes finos [18-21].

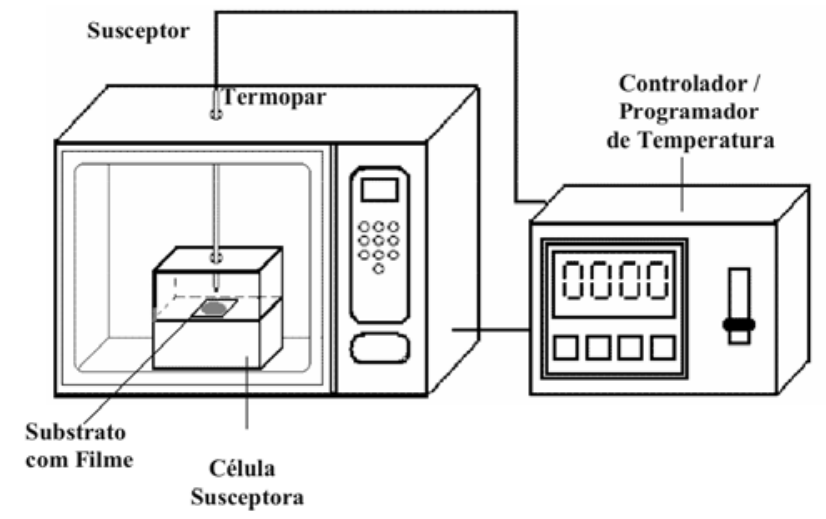

Figura 1: Configuração funcional de um forno de microondas doméstico adaptado a um controlador externo e equipado com a célula de microonda.

[Figure 1: Functional configuration of domestic microwave oven adapted with external temperature controller and a microwave cell.]

\section{MATERIAIS E MÉTODOS}

O dispositivo objeto deste trabalho consiste de um conjunto de adaptações efetuadas sobre um forno de microondas doméstico aliado a um simples aparato denominado célula susceptora conforme representação esquemática da Fig. 1. O forno de microondas usado foi um dos modelos mais simples disponíveis no mercado, no caso um CCE/M-301 de 2,45 GHz de freqüência e $900 \mathrm{~W}$ de potência. Como pode ser visto na Fig. 1 as adaptações consistiram no seguinte: a) abertura de um orifício de $3 \mathrm{~mm}$ de diâmetro sobre a parte superior da cavidade do forno para a passagem de um termopar, b) controle da magnetron por um dispositivo controlador externo, e c) adição da célula de microonda (aparato desenvolvido para este fim) no interior da cavidade do forno. O termopar que se insere pelo orifício liga-se ao controlador externo. Este, por sua vez, efetua o controle da atividade da magnetron do forno segundo o que foi previamente programado. $\mathrm{O}$ aparato que funciona como uma célula sensível a microondas se constitui na parte principal do sistema e consiste de duas peças principais: o corpo refratário e o susceptor de microondas (Fig. 1). O material usado para confecção do corpo refratário da célula foi uma mistura de óxido de alumínio e silício na proporção aproximada de 7:3, comercialmente conhecida como KAOWOOL ${ }^{\circledR} 1600$ [22]. Este material apresenta propriedade refratária e transparência às microondas, considerando-se os altos regimes de temperaturas (até $1600^{\circ} \mathrm{C}$ ) e abundância de irradiação que o aparato será submetido.

O chamado susceptor de microondas é uma pastilha densa de $\mathrm{SiC}$ que funciona como conversor de microondas em calor. Como este material apresenta propriedade de alta perda dielétrica e tempo de excitação/relaxamento de dipolos concordantes com a mudança de sentido dos dipolos das microondas de um forno de microondas (2.450.000 vezes por segundo), ele funciona como um eficiente conversor destas oscilações eletromagnéticas em calor. A pastilha de $\mathrm{SiC}$ situada na parte interna inferior da cavidade da célula susceptora é também a peça que recebe o cadinho, célula reacional ou o substrato com filme que será termicamente processado, funcionando como se fosse a resistência elétrica de um forno elétrico convencional de onde o fluxo de calor é irradiado. Assim, a célula susceptora uma vez introduzida na cavidade do forno de microondas modificado, conforme a Fig. 1, configura o novo dispositivo que pode operar com programação de aquecimento, desaquecimento e rampas à justa semelhança de um forno tubo convencional.

\section{RESULTADOS E DISCUSSÃO}

Usando o dispositivo mencionado, foram obtidos pós de óxidos metálicos não susceptíveis às microondas, bem como efetuado-se pela primeira vez o tratamento de filmes finos usando-se microondas como fonte de energia. Nesses casos, todos os materiais de partida levados a tratamento em microondas relatados foram preparados pelo método dos precursores poliméricos (Método Pechini) [20, 21].

O método Pechini [23] é um atraente método para preparação de filmes finos e pós, oferecendo algumas vantagens sobre outros métodos, tais como; mais preciso controle estequiométrico, homogeneidade, uso de solução aquosa, alta pureza e processo de baixa temperatura e baixo custo $[25,26]$. O uso deste método tem sido reportado na 
preparação de óxidos e filmes como $\mathrm{LiNbO}[25,26]$, SrTiO, $\mathrm{BaTiO}$ [27], PbTiO [28], $\mathrm{PbZr}_{0,53} \mathrm{Ti}_{0,44} \mathrm{O}_{3}$ [29] e $\mathrm{SrBi}_{2} \mathrm{Nb}_{2} \mathrm{O}_{9}$ [30]. Todos estes foram por nós tratados usando microondas e apresentaram boas características elétricas e óticas.

No caso dos pós, foram processados em microondas os puffs previamente tratados em forno convencional por $2 \mathrm{~h} \mathrm{a}$ $300{ }^{\circ} \mathrm{C}$ para efeito de eliminação de material orgânico. Para com os filmes, as resinas depositadas por spin-coating foram igualmente tratadas preliminarmente em forno convencional por 2 a 6 h a $300{ }^{\circ} \mathrm{C}$.

Entre os resultados obtidos na síntese de pós, destacase uma nova fase de óxido de alumínio dopado ao que se chamou alumina negra, dada a presença do carbono dopante inserido na rede cristalina [31]. No caso dos filmes, os resultados mais expressivos foram: crescimento epitaxial de filme fino de $\mathrm{LiNbO}_{3}$ [18], o efeito da direção do fluxo de calor sobre as propriedades elétricas do filme fino de $\mathrm{SrBi}_{2} \mathrm{NbO}_{9}$ cristalizado em forno de microondas [19], e a

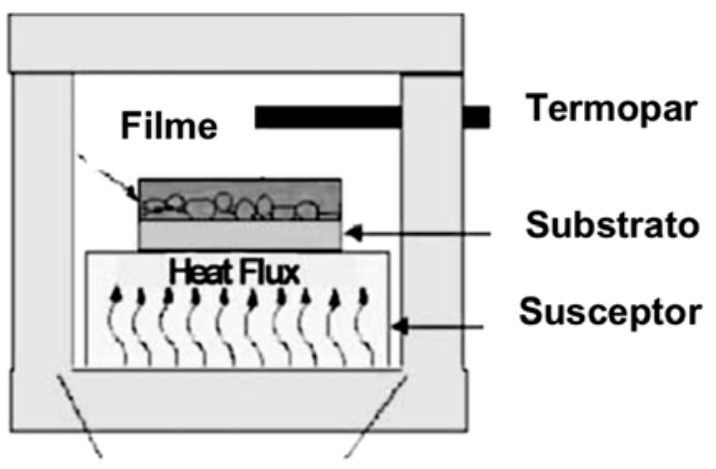

(a) Material Refratário

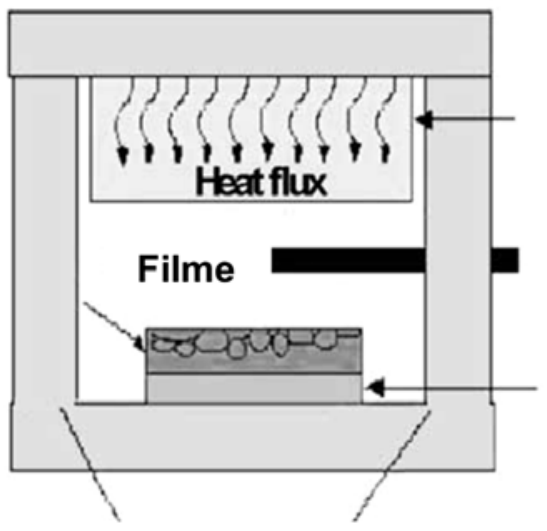

\section{Susceptor}

Termopar

\section{Substrato}

(b) Material Refratário

Figura 2: Estrutura do aparato para cristalização dos filmes finos usando microondas: (a) susceptor localizado abaixo do substrato e (b) susceptor posicionado acima do filme fino.

[Figure 2: Structure of the apparatus for crystallization of thin films using microwave: (a) susceptor placed below the substrate and (b) susceptor placed above the film.] obtenção de uma alta constante dielétrica do filme fino de $\mathrm{Pb}_{1-\mathrm{x}} \mathrm{Ba}_{\mathrm{x}} \mathrm{TiO}_{3}$ altamente orientado preparado por deposição química [32].

$\mathrm{O}$ estudo do $\mathrm{LiNbO}_{3}$ demonstrou que o processamento de filmes finos em microondas é eficiente na orientação do cristal, conferindo-lhe um crescimento epitaxial com microestrutura densa e homogênea de excelentes propriedades elétricas e óticas semelhantes às do monocristal de $\mathrm{LiNbO}_{3}$ [18]. A Tabela I mostra os índices de refração e espessura respectiva dos filmes $\mathrm{LiNbO}_{3}$ tratado por microonda comparado a outros valores encontrados na literatura e ao do simples cristal [33, 34].

$\mathrm{O}$ estudo do filme fino de $\mathrm{SrBi}_{2} \mathrm{NbO}_{9}$ revelou que o processamento de filmes em microondas usando o dispositivo descrito pode variar em função da direção do fluxo de calor que emana do susceptor, ocorrendo melhor orientação da rede quando o substrato/filme é posto sobre o susceptor de $\mathrm{SiC}$ (Fig. 2a) e menor orientação cristalina quando posto abaixo (Fig. 2b) [19].

A Fig. 3 ilustra os padrões do filme de SBN termicamente tratados em forno de microondas a $700{ }^{\circ} \mathrm{C}$ por $10 \mathrm{~min}$ com o susceptor posicionado abaixo do substrato, e os padrões do mesmo filme tratados a $700{ }^{\circ} \mathrm{C}$ por $10 \mathrm{~min}$ e $30 \mathrm{~min}$ com o susceptor posicionado acima do filme. Por comparação, os filmes de SBN foram também preparados a $700{ }^{\circ} \mathrm{C}$ por $2 \mathrm{~h}$ em um forno convencional.

Como pode ser observado na Fig. 3a, o intenso pico (008) e o pico (0010) indicam a orientação preferencial na direção c, quando o susceptor está posicionado abaixo do substrato. Não há neste caso pico de intensidade significativa correspondente a uma segunda fase. Quando o susceptor é

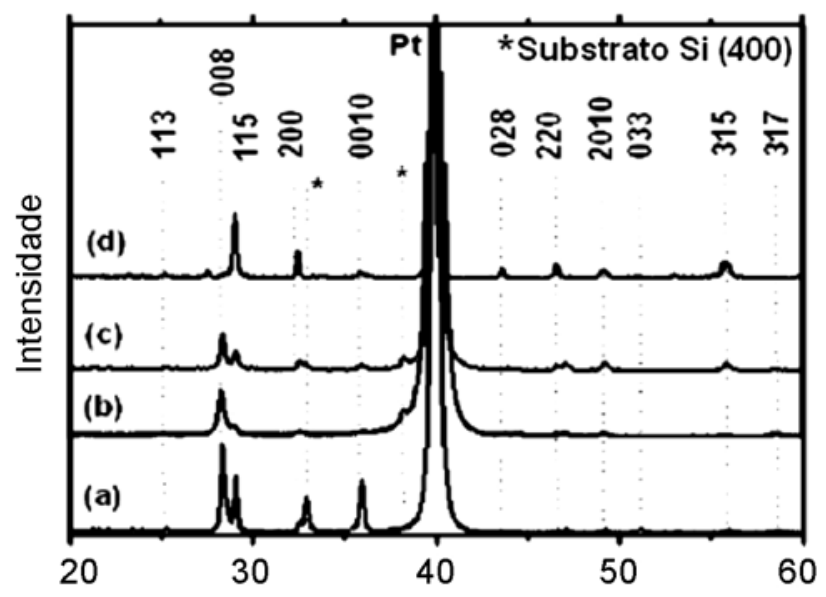

Figura 3: Padrão de DRX dos filmes de SBN termicamente tratados em forno de microondas a $700{ }^{\circ} \mathrm{C}$ : (a) com o susceptor posicionado abaixo do substrato por $10 \mathrm{~min}$, e, com o susceptor posicionado acima do filme por $10 \mathrm{~min}$ (b), $30 \mathrm{~min}$ (c) e em forno convencional (d).

[Figure 3: XRD patterns for SBN films heat-treated in microwave oven at $700^{\circ} \mathrm{C}$ : (a) with the susceptor placed below the substrate for $10 \mathrm{~min}$, with the susceptor placed above the film for (b) $10 \mathrm{~min}$ and (c) $30 \mathrm{~min}$, and (d) conventional furnace.] 
colocado acima do filme (Figs. $3 \mathrm{~b}$ e c), apresenta crescimento policristalino similar ao que ocorre com o filme é obtido em forno convencional (Fig. 3d.), embora $10 \mathrm{~min}$ seja um tempo muito curto para obtenção da fase perovisquita. Por outro lado, a fase perovisquita foi obtida quando o filme de SBN foi tratado por $30 \mathrm{~min}$.

No estudo do filme fino de $\mathrm{Pb}_{1-x} \mathrm{Ba}_{x} \mathrm{TiO}_{3}$ preparado por deposição química e orientação por microondas foi observado um excepcional acréscimo do valor da constante dielétrica desta nanoestrutura em virtude da alta orientação alcançada pelo tratamento por microondas.

A Fig. 4 mostra o padrão de difração de raios $X$ de (a) (100) substrato do $\mathrm{LaAlO}_{3}$, (b) estrutura do $\mathrm{LaNiO}_{3}$ / $\mathrm{LaAlO}_{3}$ e (c) estrutura do $\mathrm{Pb}_{0.8} \mathrm{Ba}_{0.2} \mathrm{TiO}_{3} / \mathrm{LaNiO}_{3}$ / $\mathrm{LaAlO}_{3}$. Isto pode mostrar que o eletrodo de filme fino do $\mathrm{LaNiO}_{3}$ tratado a $700{ }^{\circ} \mathrm{C}$ por 10 min encontra-se em alta orientação (100) dentro da simples fase perovisquita (a). A Fig. 4(c) mostra o filme fino de $\mathrm{Pb}_{0.8} \mathrm{Ba}_{0.2} \mathrm{TiO}_{3}$ depositado sobre a estrutura $\mathrm{LaNiO}_{3} / \mathrm{LaAlO}_{3}$, onde a intensidade dos picos (100) e (200) são tão fortes quanto os picos (101) e (110), indicando que o filme fino de $\mathrm{Pb}_{0.8} \mathrm{Ba}_{0.2} \mathrm{TiO}_{3}$ encontrase altamente orientado, apresentando somente a fase perovisquita. Portanto, ambos os filmes têm alta orientação (100) devido aos seguintes fatores; melhoria da cristalização e melhor crescimento interfacial do filme fino. Ambos foram significativamente influenciados pelo tratamento com microondas, e pela proximidade dos parâmetros de rede entre os filmes finos do $\mathrm{LaNiO}_{3}$ e do $\mathrm{Pb}_{0.8} \mathrm{Ba}_{0.2} \mathrm{TiO}_{3}$. Na Fig. 4(b) a intensidade do pico (100) do substrato é muito mais fraca do que a do pico (200) encontrado. Isto está em concordância com a ordem dos picos observados no substrato puro na Fig. 4(a). A Fig. 4(c) não apresenta no geral pico relativo ao substrato. Neste caso pode-se considerar que a espessura da estrutura intermediaria $\mathrm{Pb}_{08} \mathrm{Ba}_{02} \mathrm{TiO}_{3} / \mathrm{LaNiO}_{3}$ tenha algum papel na explicação disto.

A Fig. 5 mostra que a freqüência depende da constante dielétrica da perda dielétrica do filme fino do $\mathrm{Pb}_{0.8} \mathrm{Ba}_{0.2} \mathrm{TiO}_{3}$ suportado sobre o eletrodo de $\mathrm{LaNiO}_{3}$. A constante dielétrica mostra uma discreta diminuição e perda dielétrica que cresce lentamente com o crescimento da freqüência. $\mathrm{O}$ valor da constante dielétrica a uma freqüência de $100 \mathrm{kHz}$ é 1804 . Isto é maior do que a que foi reportada, em torno de 118, para filmes finos de $\mathrm{Pb}_{0.8} \mathrm{Ba}_{02} \mathrm{TiO}_{3}$ derivados de sol-gel e depositados sobre eletrodos de platina [35]. Adicionalmente, a constante dielétrica obtida neste trabalho também é muito maior do que as de outros filmes depositados sobre eletrodos de $\mathrm{LaNiO}_{3}$ reportados na literatura [36, 37]. Bao et al. [38] reportaram um valor de constante dielétrica de 1468 a uma freqüência de $1 \mathrm{kHz}$ com polarização remanescente e campo coercitivo em torno de $4,24 \mathrm{mC} / \mathrm{cm} 2$ e $23,2 \mathrm{kV} / \mathrm{cm}$ respectivamente, para um filme fino de $\mathrm{Pb}_{0.75} \mathrm{Ba}_{0.25} \mathrm{TiO}_{3}$ preparado por sol-gel sobre eletrodo suporte de $\mathrm{LaNiO}_{3}$. Wang et al. [39] relatou um valor de constante dielétrica em torno de 650 a uma freqüência de $1 \mathrm{kHz}$ e polarização remanescente e campo coercitivo de $10,8 \mathrm{mC} / \mathrm{cm}^{2}$ e $96 \mathrm{kV} / \mathrm{cm}$ respectivamente, para filme fino de
$\mathrm{Ba}_{0.9} \mathrm{Sr}_{0.1} \mathrm{TiO}_{3}$ sobre eletrodo suporte de $\mathrm{LaNiO}_{3}$. Note-se que o valor da constante dielétrica para o filme fino de $\mathrm{Pb}_{0.8} \mathrm{Ba}_{0.2} \mathrm{TiO}_{3}$ depositado sobre eletrodo de $\mathrm{LaNiO}_{3}$ é mais alta do que a de filmes depositados diretamente sobre platina ou $\mathrm{LaNiO}_{3}$ preparado por outras técnicas. A melhoria observada pode se dever ao aumento entre as interfaces dos filmes finos de $\mathrm{Pb}_{0.8} \mathrm{Ba}_{0.2} \mathrm{TiO}_{3}$ e o eletrodo de $\mathrm{LaNiO}_{3}$, que poderia resultar na formação de uma estrutura de $\mathrm{Pb}_{0.8} \mathrm{Ba}_{0.2} \mathrm{TiO}_{3} / \mathrm{LaNiO}_{3} /$ $\mathrm{LaAlO}_{3}$ altamente orientada, bem como de um filme fino de $\mathrm{Pb}_{0.8} \mathrm{Ba}_{0.2} \mathrm{TiO}_{3}$ com grande tamanho de grão. Isto dá suporte à idéia de que o tratamento em forno de microondas impede a

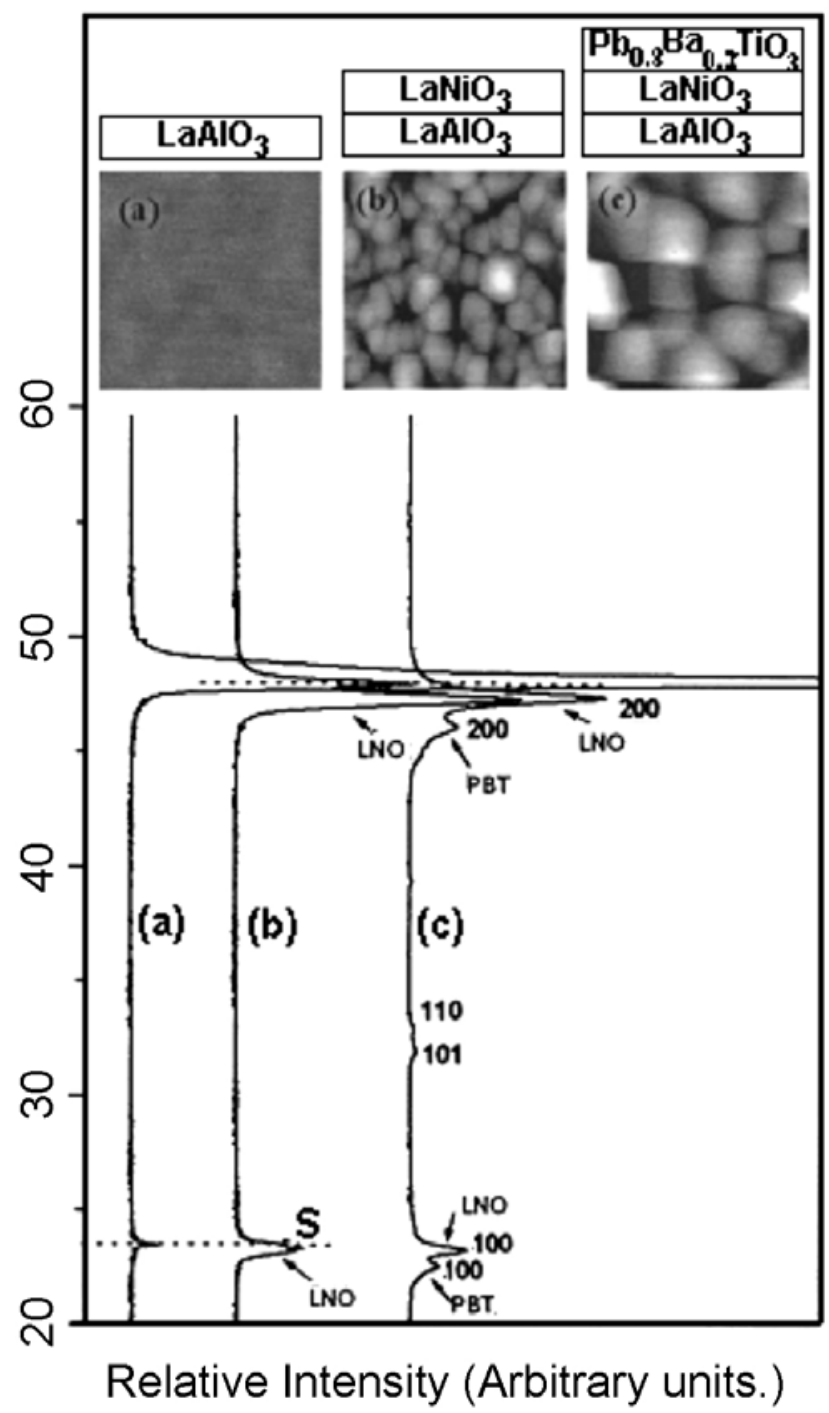

Figura 4: Difratogramas de raios $\mathrm{X}$ de (a) substrato do the $\mathrm{LaAlO}_{3}$ (100); (b) filme fino de $\mathrm{LaNiO}_{3}$ sobre substrato de $\mathrm{LaAlO}_{3}$ (100); e (c) do filme fino de $\mathrm{Pb}_{08} \mathrm{Ba}_{02} \mathrm{TiO}_{3}$ sobre $\mathrm{LaNiO}_{3} / \mathrm{LaAlO}_{3}(100)$. Imagens de MFA inseridas (131 $\mu \mathrm{m}$ ) (a) o substrato; (b) o filme de $\mathrm{LaNiO}_{3}$ e (c) o filme fino $\mathrm{Pb}_{0.8} \mathrm{Ba}_{0.2} \mathrm{TiO}_{3} . \mathrm{S}=$ pico do substrato.

[Figure 4: X-ray diffraction patterns of (a) the $\mathrm{LaAlO}_{3}(100)$ substrate; (b) $\mathrm{LaNiO}_{3}$ thin film on the $\mathrm{LaAlO}_{3}$ (100) substrate; and (c) the $\mathrm{Pb}_{0.8} \mathrm{Ba}_{0.2} \mathrm{TiO}_{3}$ thin film on $\mathrm{LaNiO}_{3} / \mathrm{LaAlO}_{3}(100)$. Inset shows the AFM micrographs $(131 \mu \mathrm{m})$ of the (a) substrate; (b) LaNiO3 thin film; and (c) $\mathrm{Pb}_{0.8} \mathrm{Ba}_{0.2} \mathrm{TiO}_{3}$ thin film. $\mathrm{S}=$ Substrate peak.] 
formação de uma baixa constante dielétrica da rede do filme fino/eletrodo de interface, que é a principal causa de baixos valores de constante dielétrica relatadas em muitos filmes finos ferroelétricos [38].

Os resultados de alta orientação e melhoria das propriedadeselétricaseópticasforamatribuídosàcombinação do uso de método de química leve com o processamento por microondas utilizando-se o aparato descrito neste trabalho. A alta orientação e constante dielétrica verificada no filme de $\mathrm{Pb}_{1-\mathrm{x}} \mathrm{Ba}_{\mathrm{x}} \mathrm{TiO}_{3}$ potencializou as possibilidades de aplicação deste material como dispositivos eletrônicos de supermemórias de computador aplicável à indústria eletrônica [32].

Anteriormente ao surgimento desta nova técnica de processamento, o uso de microondas na síntese química convencionou-se por ser feito a partir de sistemas reacionais que necessariamente contivessem componentes susceptíveis. Esta forma de raciocínio pressupunha a existência de pelo menos um elemento diretamente participante da síntese ou processo com o qual as microondas pudessem interagir. Ocorre, entretanto, que apesar do conceito correto, tal modos operandis impossibilitava outras aplicações das microondas em processamento de materiais, principalmente quando os sistemas não possuíam massa suficiente para interagir significativamente com as microondas ou quando se dispunha de sistemas reacionais sólidos, líquidos ou gasosos não susceptíveis. A perspectiva de não reter o elemento susceptível, no caso o $\mathrm{SiC}$, circunscrito aos elementos diretamente participantes do processo, possibilitou a ampliação praticamente sem limites do número de materiais que agora podem ser tratados usando o dispositivo descrito.

$\mathrm{O}$ desenvolvimento do referido aparato pode resultar em outras vantagens além das propriedades adicionais obtidas pelo processamento. Uma dessas vantagens decorre do seguinte: enquanto em um forno de microondas doméstico a temperatura máxima não ultrapassa $250{ }^{\circ} \mathrm{C}$ durante $\mathrm{o}$ cozimento de alimentos ou um pouco mais sobre a maioria de materiais susceptíveis, com este novo dispositivo é possível alcançar temperaturas de até $1400{ }^{\circ} \mathrm{C}$ com o susceptor indo rapidamente a rubro intenso. Mesmo considerando que as dimensões da célula são reduzidas em relação às dimensões de um forno convencional, o benefício do seu uso aparece no razoável valor final do dispositivo e nas amplas possibilidades de aplicação na pesquisa científica.

Extrapolações efetuadas sobre a idéia apresentada poderão certamente render outros modelos diferentes de células susceptíveis às microondas e fornos acoplados a essas células adequados a outros processos e reações.

Em relação à interação microonda/material, os motivos pelos quais ocorrem ganhos de energia/tempo em muitos processos físico-químicos ainda não se encontram bem estabelecidos. Entretanto, pode-se identificar que alguns ganhos auferidos em sínteses e processos na presença de microondas ocorrem pelo menos pelo seguinte: melhor orientação de redes cristalinas em virtude do efeito de fluxo

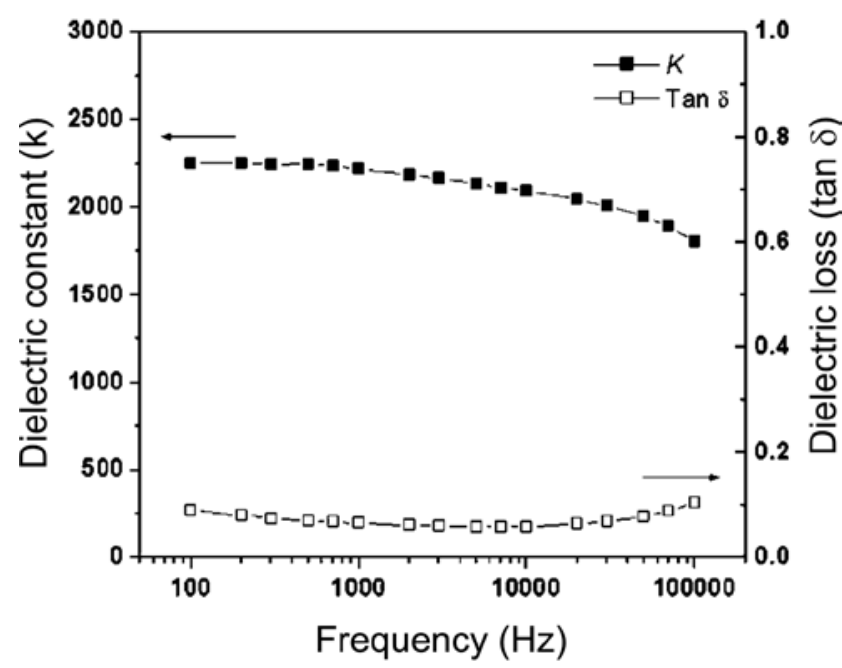

Figura 5: Dependência da freqüência da constante dielétrica e perda dielétrica do filme fino de $\mathrm{Pb}_{0.8} \mathrm{Ba}_{0.2} \mathrm{TiO}_{3}$ com a configuração $\mathrm{Au} / \mathrm{PBT} / \mathrm{LaNiO}_{3} / \mathrm{LaAlO}_{3}(100)$.

[Figure 5: Frequency dependence of the dielectric constant and dielectric loss of the $\mathrm{Pb}_{0.8} \mathrm{Ba}_{0.2} \mathrm{TiO}_{3}$ thin film with a $\mathrm{Au} / \mathrm{PBT} / \mathrm{LaNiO}_{3}$ $/ \mathrm{LaAlO}_{3}$ (100) configuration.]

de calor vindo do susceptor do aparato [18] e/ou cisões/ formações preferenciais de ligações polares susceptíveis aos dipolos magnéticos das microondas.

Um fenômeno que ora encontra-se sendo investigado por E. Longo et al. é um possível efeito não térmico exercido sobre a orientação da rede cristalina de óxidos metálicos, devido ao acoplamento do dipolo de moléculas ou conjuntos de átomos com o dipolo das microondas. Por outro lado, ordenações mais rápidas de redes cristalinas podem ser observadas quando o material atinge temperaturas além da ordinária (acima de $600{ }^{\circ} \mathrm{C}$ ). Para esta hipótese, ainda que o material de partida seja não susceptível e desconsidere-se o efeito do fluxo de calor, pode ocorrer maior mobilidade no interior do material sob processamento em decorrência da interação mais e mais pronunciada entre os dipolos elétricos do material e as microondas.

$\mathrm{O}$ fato da temperatura de muitos materiais, ao ultrapassarem níveis de temperatura ordinária, aumentar sensivelmente as vibrações atômicas, certamente implica no incremento da susceptibilidade desses materiais às microondas, proporcionando energia térmica adicional. Nesta hipótese, o próprio material aquecido, ora comportando-se como susceptor, passa a contribuir com uma parcela de calor adicional a ser usado durante o processo de síntese ou do tratamento que está sendo processado.

Sobre o aspecto construtivo do aparato, a escolha da manta refratária deveu-se às suas propriedades de transparência à microonda, isolamento térmico e alto ponto de fusão combinados em um mesmo material. Obviamente, qualquer outro material que possua características similares, poderia ser utilizado convenientemente na construção de células com semelhantes fins. Entretanto, é possível pressupor células 
Tabela I - Índice de refração $(\lambda=632,8 \mathrm{~nm})$ e espessura do filme de $\mathrm{LiNbO}_{3}$ processado em forno de microondas comparados a valores encontrados na literatura.

[Table 1-Refractive index (at $\lambda=632.8 \mathrm{~nm}$ ) and thickness of the $\mathrm{LiNbO}_{3}$ films processed in the microwave oven and values taken from the literature.]

\begin{tabular}{ccccc}
\hline Referência & $\begin{array}{c}\text { Método de } \\
\text { Obtenção }\end{array}$ & $\begin{array}{c}\text { Temperatura } \\
\left({ }^{\circ} \mathrm{C}\right)\end{array}$ & $\begin{array}{c}\text { Índice } \\
\text { de } \\
\text { Reflexão }\end{array}$ & $\begin{array}{c}\text { Espessura } \\
(\mathrm{nm})\end{array}$ \\
\hline Este trabalho & Pechini & 450 & 1,91 & 143 \\
Este trabalho & Pechini & 550 & 2,16 & 235 \\
Este trabalho & Pechini & 650 & 1,97 & 257 \\
Nashimoto [39] & Sol-gel & 700 & 2,24 & 90 \\
{$[40]$} & Sol-gel & 600 & 2,16 & 108 \\
{$[41]$} & PLD & 750 & 2,24 & - \\
{$[42]$} & Pechini & 550 & 2,15 & 71 \\
{$[43]$} & MOCVD & 700 & 2,26 & $150-170$ \\
{$[34]$} & r. f. sputtering & 500 & 2,30 & - \\
{$[33,34]$} & monocristal & - & 2,28 & - \\
\hline
\end{tabular}

susceptoras não necessariamente refratárias ou transparentes tendo em vista a conveniência da sua aplicação. Sob estes aspectos, a própria pesquisa de materiais e compósitos convenientes à construção de células susceptoras, visando diversas aplicações pode se constituir em um interesse de pesquisa de per se.

\section{CONCLUSÕES}

O uso da tecnologia de microondas como fonte de energia para síntese e processamento de materiais tem se mostrado de relevante importância para a química e outras ciências dos materiais.

O uso do aparato citado possibilitou pela primeira vez a utilização de microondas na preparação e ordenação da rede cristalina de filmes finos e o uso de fornos domésticos de microondas na síntese e processamento de materiais não susceptíveis. Este fato ampliou praticamente a um número sem limites os materiais e substâncias que podem ser sintetizadas e processadas usando microondas como fonte de energia.

De igual forma, o conjunto de novos resultados obtidos pela interação calor/microonda/material, tem feito com que seja viável a continuação e o estudo de outros materiais por esta técnica, bem como encorajado o desenvolvimento de aparatos similares que se preste a obtenção de novos resultados.

\section{REFERÊNCIAS}

[1] R. A. Abramovich, Org. Prep. Proced. Int. 23 (1991) 683.

[2] R. Gedye, F. Smith, K. Westaway, H. Ali, L. Baldisera, L. Laberge, J. Rousell, Tetrahedron Lett. 27 (1986) 279.

[3] R. J. Giguere, T. L. Bray, S. M. Duncan, G. Majetich, Tetrahedron Lett. 27 (1986) 4945.

[4] C. R. Strauss, R. W. Trainor, Aust. J. Chem. 48 (1995) 1665.
[5] A. Loupy, A. Petit, D. Bunnet-Delpon, J. Fluor. Chem. 75 (1995) 215.

[6] G. Link, V. Ivanov, S. Paranin, V. Khrustov, R. Böhme, G. Muller, G. Schumacher, M. Thumm, A. Weisenburger, Mater. Res. Soc. Symp. Proc. 430 (1996) 157.

[7] Y. V. Bykov, A. G. Eremeev, A. G. Holoptsev, V. V. Odemer, C. Rachkovskii, A. I. Kleissl, H. J. R. Ceram. Trans. 80 (1997) 321.

[8] Q. Zhang, J. Luo, E. Vileno, S. L. Suib, Chem. Mater. 9 (1997) 2090.

[9] T. Gerdes, M. Willert-Porada, K. Rödiger, K. Dreyer, Mater. Res. Soc. Symp. Proc. 430 (1996) 175.

[10] F. Selmi, F. Guerin, X.D. Xu, V. K. Varadan, V. V. Varadan, S. Komarneni, Mater. Lett. 12 (1992) 424.

[11] S. A. Nightingale, H. K. Worner, D. P. Dunne, J. Am. Ceram. Soc. 80 (1997) 394.

[12] D. M. P. Mingos, D. R. Baghurst, Chem. Soc. Rev. 20 (1991) 1.

[13] S. Caddick, Tetrahedron 51 (1995) 10403.

[14] A. K. Bose, B. K. Banik, N. Lavlisnkaia, M. Jayaraman, M. S. Manhas, Chemthec 27 (1997) 18.

[15] R. S. Varma, Green Chem. 1 (1999) 43.

[16] A. C. R. N. Barboza, C. M. S. Cruz, M. B. Graziani, M.

C. F. Lorenzetti, E. Sabadini, Quím. Nova 24, 6 (2001) 901.

[17] K. J. Rao, B. Vaidhyanathan, M. Ganguli, P. A. Ramakrishnan, Chem. Mater. 11 (1999) 882.

[18] N. S. L. S. Vasconcelos, J. S. Vasconcelos, V. Bouquet, S. M. Zanetti1, E. R. Leite, E. Longo, M. I. Bernardi, A. Perrin, M. Guilloux-Viry, J. A. Varela, Thin Solid Films 436 (2003) 213.

[19] J. S. Vasconcelos, N. S. L. S. Vasconcelos, S. M. Zanetti, E. R. Leite, J. A. Varela, E. Longo, Appl. Surf. Sci. 225 (2004) 156.

[20] N. S. L. S. Vasconcelos, J. S. Vasconcelos, V. Bouquet, S. M. Zanetti1, E. R. Leite, E. Longo, M. I. Bernardi, A. 
Perrin, M. Guilloux-Viry, J. A. Varela, Cerâmica 50, 314 (2004) 128.

[21] J. S. Vasconcelos, N. S. L. S. Vasconcelos, S. M. Zanetti1, J. W. Gomes, E. Longo, E. R. Leite, J. A. Varela, Cerâmica 49, 309 (2003) 61.

[22] Thermal Ceramics, Catalogue Morganite do Brasil, disponível em http://www.thermalceramics.com/ morganitethermal.com.br, acessado em maio 2005.

[23] M. P. Pechini,US Patent N. 3330697 (1967).

[24] V. Bouquet, M. I. B.Bernardi, S. M. Zanetti, E. Longo, E. R. Leite, J. A. Varela, M. Guilloux-Viry, A. Perrin, J. Mater. Res. 15 (2000) 2446.

[25] F. M. Pontes, E. R. Leite, E. Longo, J. A. Varela, P. S. Pizani, C. E. M. Campos, F. Lanciotti, Adv. Mater. Opt. Electron. 10 (2000) 1.

[26] V. Bouquet, E. R. Leite, E. Longo, J. A. Varela, Key Eng. Mater. 132 (1997) 1143.

[27] F. M. Pontes, E. R. Leite, E. J. H. Lee, E. Longo, J. A. Varela, Thin Solid Films 385 (2001) 260.

[28] E. R. Leite, F. M. Pontes, E. C. Paris, C. A. Paskocimas, E. Lee, E. Longo, P. S. Pizani, J. A.Varela, V. Mastelaro, Adv. Mater. Opt. Electron. 10 (2000) 1.

[29] M. S. J. Nunes, E. R. Leite, F. M. Pontes, N. M. Duboc, E. Longo, J. A. Varela, Mater. Lett. 49 (2001) 365.

[30] S. M. Zanetti, E. B. Araujo, E. R. Leite, E. Longo, J. A. Varela, Mater. Lett. 40 (1999) 33.

[31] J. S. Vasconcelos, R. C. Lima, N. S. L. S. Vasconcelos, C. A. Paskocimas, E. R. Leite, J. A. Varela, E. Longo,
"Aparato e método para obtenção de alumina gama de cor preta usando forno de microondas doméstico", Patente Requerida INPI-BR (2004).

[32] F. M. Pontes, E. R. Leite, G. P. Mambrini, M. T. Escote, and E. Longo, Appl. Phys. Lett. 84 (2004) 2.

[33] N. H. Hur, Y. K. Park, D. H. Won, K. No, J. Mater. Res. 9 (1994) 980.

[34] S. Schwyn, H. W. Lehmann, R. Widmer, J. Appl. Phys. 72 (1992) 1154.

[35] N. V. Giridharan, R. Jayavel, Mater. Lett. 52 (2002) 57.

[36] D. Bao, N. Mizutani, X. Yao, L. Zhang, Appl. Phys. Lett. 77 (2000) 1041

[37] R. R. Das, P. Bhattacharya, R. S. Katiyar, A. S. Bhalla, J. Appl. Phys. 92 (2002) 6160.

[38] D. Bao, N. Wakiya, K. Shinozaki, N. Mizutani, X. Yao, Appl. Phys. Lett. 78 (2001) 3286.

[39] K. Nashimoto, H. Moriyama, E. Osakabe, Jpn. J. Appl. Phys. 35 (1996) 4936.

[40] S. Ono, O. Bose, W. Unger, Y. Takeichi, S. Hirano, J. Am. Ceram. Soc. 81 (1998) 1749.

[41] P. Aubert, G. Garry, R. Bisar, L. Garcia, Appl. Surf. Sci. 86 (1995) 144.

[42] V. Bouquet, M. I. B. Bernardi, S. M. Zanetti, E. Longo, E. R. Leite, J. A. Varela, M. Guilloux Viry, A. Perrin, J. Mater. Res. 15 (2000) 2446.

[43] S. Y. Lee, R. S. Feigelson, J. Cryst. Growth 186 (1998) 594.

(Rec. 25/05/2005, Ac. 30/09/2005) 